\title{
Coping With COVID-19: An Online Educational Course
}

Sue Hall, MD

A viral pandemic. A racial pandemic within the viral pandemic. Is mental illness the next inevitable pandemic? Many think so.

The COVID-19 pandemic has heaped a variety of psychosocial stresses on pregnant people, new parents, and frontline medical providers, and has had a disproportionate effect on disadvantaged communities and people of color.

The National Perinatal Association has joined with the NICU Parent Network to create My NICU Network-My Perinatal Network to provide online continuing education on the topic of providing psychosocial support to parents, patients, and staff. The newest offering, "Coping with COVID-19," which is "just in time" education, is a short (3-course) program of educational courses to give NICU providers more knowledge and skills about how to support patients' and NICU staff's emotional well-being during the COVID-19 pandemic. As stated in the New England Journal of Medicine: "Given that most COVID-19 cases will be identified and treated in health care settings by workers with little to no mental health training, it is imperative that assessment and intervention for psychosocial concerns be administered in those settings" (Pfefferbaum and North, 2020). Our goal, therefore, is to provide frontline NICU and maternity providers with an understanding of trauma-informed care, how to identify patient populations at greatest risk of experiencing adverse effects such as Perinatal Mood and Anxiety Disorders as a result of the pandemic, and how to provide psychosocial supports to mitigate the potential that these stresses could evolve into more serious problems.

"Our goal, therefore, is to provide frontline NICU and maternity providers with an understanding of traumainformed care, how to identify patient populations at greatest risk of experiencing adverse effects such as Perinatal Mood and Anxiety Disorders as a result of the pandemic, and how to provide psychosocial supports to mitigate the potential that these stresses could evolve into more serious problems."

This program for NICU providers (physicians, nurses, nurse practitioners, social workers, educators, therapists, etc.) consists of 3 courses:

1- Giving Birth During the COVID-19 Pandemic: Trauma-in-

\author{
formed Care to Support Patients, Families, and Staff;
}

2- Communication Skills for NICU Staff; and

3- Providing Emotional Support to NICU Parents.

The COVID-19 course reviews the impact that societal disruptions and social determinants of health have had on families' well-being and on the pronounced racial disparities that the pandemic has brought to light. It explores the anxieties which practitioners may expect to see among pregnant patients and new parents, including the distress parents are feeling about the potential and sometimes required separation from their babies after birth. The topic of staff distress related to working during the pandemic is also addressed in detail, with mitigation strategies proposed.

"The courses are available for free (nocredit) thanks to a grant that the National Perinatal Association has been awarded from the Health Resources and Services Administration of the US Department of Health and Human Services, through the University of North Carolina at Chapel Hill."

The courses are available for free (no-credit) thanks to a grant that the National Perinatal Association has been awarded from the Health Resources and Services Administration of the US Department of Health and Human Services, through the University of North Carolina at Chapel Hill. Learners are able to take the COVID-19 course only, or all 3 of the courses to get a broader foundation in both trauma-informed care and providing emotional support. If CEU/CMEs are desired, nominal fees will apply, although the COVID-19 course will remain free. All courses are clinically relevant, evidence-based, story-driven, trauma-informed, resource-

"Interested learners can also choose to take our more extensive program of 7 courses for NICU staff called "Caring for Babies and Their Families: Providing Psychosocial Support in the NICU;" maternity providers can take the new 6-course program called "Caring for Patients and Their Families: Providing Psychosocial Support During Pregnancy, Labor \& Delivery." 
rich, and family-centered, and include the patient/parent "voice."

Interested learners can also choose to take our more extensive program of 7 courses for NICU staff called "Caring for Babies and Their Families: Providing Psychosocial Support in the NICU;" maternity providers can take the new 6 -course program called "Caring for Patients and Their Families: Providing Psychosocial Support During Pregnancy, Labor \& Delivery." Information about all programs, and all included courses (which can be taken individually), are available on our websites at www.myperinatalnetwork. org or www.myperinatalnetwork.com.

\section{Reference:}

Pfefferbaum, B, and CS North. 2020. "Mental Health and the Covid-19 Pandemic." New England Journal of Medicine epub ahead of print. https://www.nejm.org/doi/full/10.1056/ NEJMp2008017.

The author has no conflicts to disclose

\section{NT}

Corresponding Author
Sue L. Hall, MD, MSW, FAAP
Neonatologist,
St. John's Regional Medical Center,
Oxnard, CA,
suehallmd@gmail.com

\section{FREE ONLINEEDUCATION}
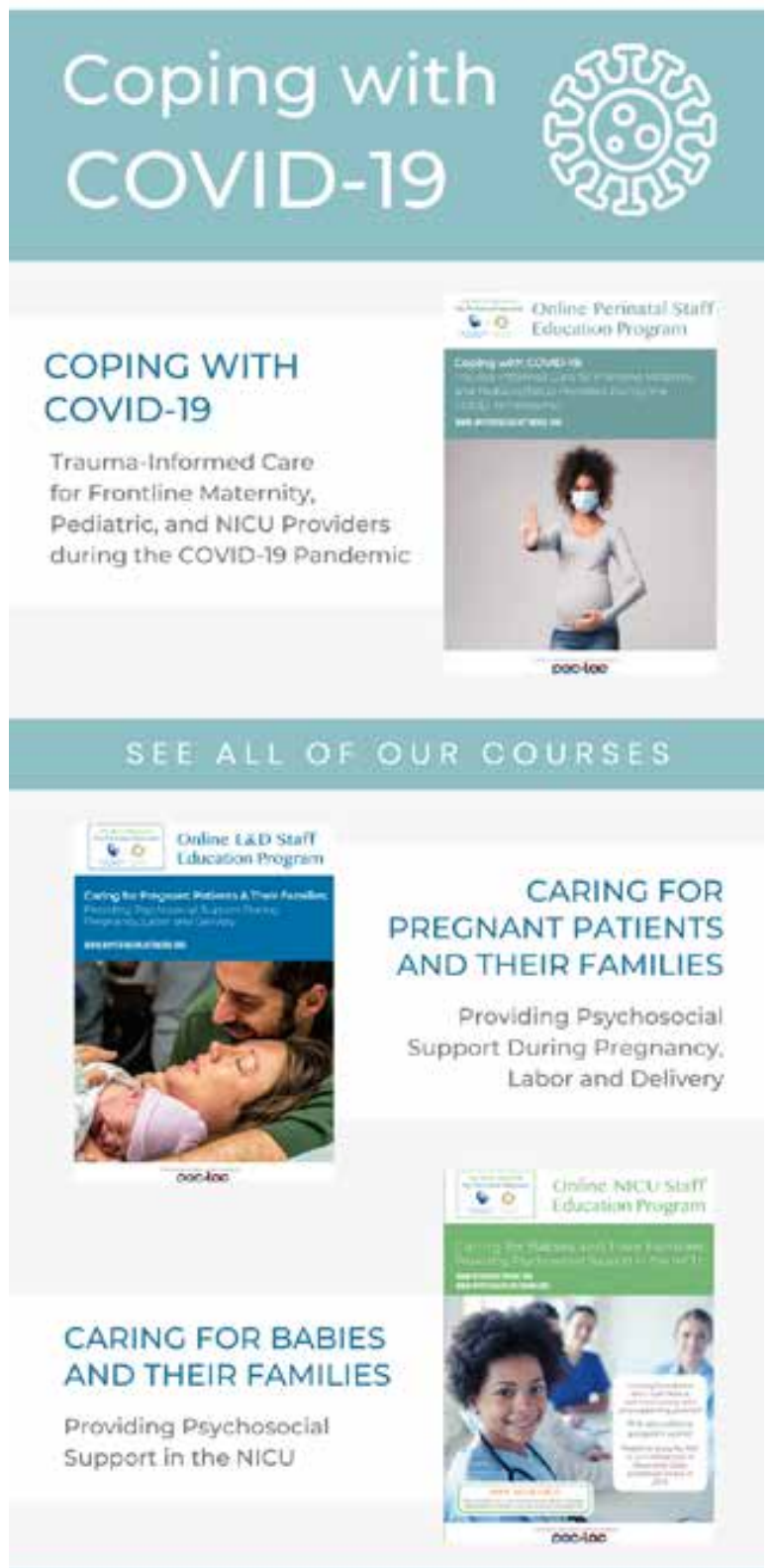

WWW.MYPERINATALNETWORK.ORG

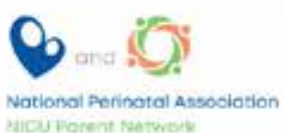

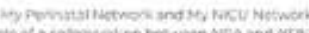

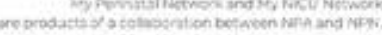

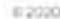

\section{THE \\ BRETT TASHMAN FOUNDATION}

The Brett Tashman Foundation is a 501@(3) public charity. The mission of the Foundation is to find a cure for Desmoplastic Small Cell Round Tumors (DSRCT). DSRCT is an aggressive pediatric cancer for which there is no cure and no standard treatment. 100 percent of your gift will be used for research. There is no paid staff. To make your gift or for more information, go to "TheBrettTashmanFoundation.org" or phone (909) 981-1530. 